\title{
Current-Voltage Temperature characteristics of Au/n-Ge (100) Schottky diodes
}

\author{
Albert Chawanda ${ }^{1,2, *}$, Wilbert Mtangi ${ }^{2}$, Francois D Auret ${ }^{2}$, Jacqueline Nel ${ }^{2}$, \\ Cloud Nyamhere ${ }^{3}$, Mmantsae Diale ${ }^{2}$ \\ ${ }^{1}$ Midlands State University, Bag 9055 Gweru, Zimbabwe \\ ${ }^{2}$ University of Pretoria, 0002 Pretoria, South Africa \\ ${ }^{3}$ Nelson Mandela Metropolitan University, Port Elizabeth, South Africa
}

\begin{abstract}
The variation in electrical characteristics of $\mathrm{Au} / \mathrm{n}-\mathrm{Ge}$ (100) Schottky contacts have been systematically investigated as a function of temperature using current-voltage $(I-V)$ measurements in the temperature range $140-300 \mathrm{~K}$. The $I-V$ characteristics of the diodes indicate very strong temperature dependence. While the ideality factor $n$ decreases, the zerobias Schottky barrier height $(\mathrm{SBH})\left(\Phi_{B}\right)$ increases with increasing temperature. The $I-V$ characteristics are analyzed using the thermionic emission (TE) model and the assumption of a Gaussian distribution of the barrier heights due to barrier inhomogeneities at the metal-semiconductor interface. The zero-bias barrier height $\Phi_{B}$ versus $1 / 2 \mathrm{kT}$ plot has been used to show evidence of a Gaussian distribution of barrier heights and values of $\bar{\Phi}_{B}=0.615 \mathrm{eV}$ and standard deviation $\sigma_{s 0}=0.0858 \mathrm{eV}$ for the mean barrier height and zero-bias standard deviation have been obtained from this plot, respectively. The Richardson constant and the mean barrier height from the modified Richardson plot were obtained as $1.37 \mathrm{Acm}^{-2} \mathrm{~K}^{-2}$ and $0.639 \mathrm{eV}$, respectively. This Richardson constant is much smaller than the reported of $50 \mathrm{Acm}^{-2} \mathrm{~K}^{-2}$. This may be due to greater inhomogeneities at the interface.
\end{abstract}

Keywords: Schottky contacts, current-voltage-temperature, Schottky barrier height, Gaussian distribution, inhomogeneities

\footnotetext{
* Corresponding author. Tel.:+27 12420 3508, +26354260 525; fax: +27 123625288.

E-mail address: albert.chawanda@up.ac.za
} 


\section{Introduction}

Metal-semiconductor (MS) contacts are the most widely used rectifying contacts in the electronic industry. The performance and reliability of a Schottky diode (SD) is drastically influenced by the interface quality between the deposited metal and semiconductor surface [1]. Therefore, MS interfaces are an essential part of virtually all semiconductor electronic and optoelectronic devices. The Schottky barrier height (SBH) is one of the most interesting properties of a MS interface as it controls the electron transport across the MS interface, and is of vital importance to the successful operation of any semiconductor device [2]. It is often found that the current-voltage $(I-V)$ characteristics of MS contacts usually deviate from the ideal thermionic emission (TE) current model [3]. Analysis of the $I-V$ characteristics of Schottky barrier diodes (SBDs) based on TE theory usually reveals an abnormal decrease in the barrier height and an increase in the ideality factor with decreasing temperature $[4,5]$. The standard TE theory fails to explain this result as it expects the SBH variation to be controlled only by the variation of band gap with temperature [6]. SBH in Schottky contacts is likely to be a function of the atomic structure, and the atomic inhomogeneities at MS interface which are caused by grain boundaries, defects, multiple phases, etc. [7]. Contaminants due to undesirable reaction products at the MS interface may act directly to introduce inhomogeneties or they may simply promote inhomogeneity through the generation of defects [8]. Schottky diodes with low SBH have found applications in devices operating at cryogenic temperatures as infrared detectors and sensors in thermal imaging $[9,10,11,12]$. Therefore, analysis of $I-V$ characteristics of Schottky diodes at room temperature only does not give detailed information about their conduction process or the nature of barrier formation at the MS interface. The temperature dependence of the $I-V$ characteristics allows us to understand different aspects of conduction mechanisms [13]. 
To the best of our knowledge there has been no report on the electrical transport characteristics of germanium (Ge) Schottky contacts at low temperatures. Therefore an attempt has been made to study the current transport characteristics of Au/n-Ge (100) Scottky diodes in the temperature range $140-300 \mathrm{~K}$. In this study, the $I$ - $V$ characteristics of $\mathrm{Au} / \mathrm{n}-\mathrm{Ge}$ (100) Schottky diodes were measured in the $140-300 \mathrm{~K}$ temperature range. The temperature dependent barrier characteristics of $\mathrm{Au} / \mathrm{n}-\mathrm{Ge}$ (100) contact were interpreted on the basis of a thermionic emission mechanism with Gaussian spatial distribution of the barrier heights around a mean value due to barrier height inhomogeneities prevailing at the MS interface.

\section{Experimental procedures}

Schottky barrier diodess were fabricated on Sb-doped (100) n-type Ge substrate with doping concentration of $2.5 \times 10^{15} \mathrm{~cm}^{3}$. The substrate was sequentially degreased with organic solvents like trichloroethylene, acetone and methanol by ultrasonic agitation for 5 minutes in each stage followed by rinsing in deionized water. The native oxide on the surface was etched in a mixture of $\mathrm{H}_{2} \mathrm{O}_{2}(30 \%): \mathrm{H}_{2} \mathrm{O}$ (1:5) for 1 minute. After rinsing in deionized water the sample was blown dry using $\mathrm{N}_{2}$. Immediately after cleaning the sample was inserted into a vacuum chamber where $100 \mathrm{~nm}$ thick $\mathrm{AuSb}(0.6 \%)$ was deposited by resistive evaporation as a back ohmic contact, followed by annealing at $350^{\circ} \mathrm{C}$ in $\mathrm{Ar}$ ambient for 10 minutes to minimize the ohmic contact's resistivity. Before contacts deposition, the sample was again chemically cleaned as described above. Au Schottky contacts were deposited by vacuum resistive evaporation through a mechanical mask. These contacts were deposited under vacuum with a pressure below $10^{-6}$ Torr. The contacts were $(0.60 \pm 0.05) \mathrm{mm}$ in diameter and $30 \mathrm{~nm}$ thick. Thickness of the contacts was measured during deposition by an INFICON XTC 751-001-G1 crystal thickness monitor. After contact fabrication, current- 
voltage $(I-V)$ measurements were performed in a He cryostat in the temperature range $140-$ $300 \mathrm{~K}$

\section{Results and discussions}

\subsection{The current-voltage characteristics as a function of temperature}

The $I-V$ relation for a MS diode, based on the TE theory can be expressed as $[1,14]$

$$
I=I_{s} \exp \left(\frac{q V}{n k T}\right)\left[1-\exp \left(-\frac{q V}{k T}\right)\right]
$$

where $I$ is the measured current, $V$ is the applied voltage, $q$ is the electronic charge, $n$ is the ideality factor that describes the deviation from the ideal diode equation for reverse bias as well as forward bias, $k$ is the Boltzmann's constant, $T$ is the absolute temperature in Kelvin, $I_{s}$ is the saturation current derived from the straight line intercept of $\ln I$ at zero-bias and is given by

$$
I_{s}=A A^{*} T^{2} \exp \left(-\frac{q \Phi_{B}}{k T}\right)
$$

where $A$ is the $\mathrm{SD}$ area, $A^{*}$ is the effective Richardson constant and $\Phi_{B}$ is the apparent barrier height at zero-bias, which can be obtained from Eq. (2)

$$
\Phi_{B}=\frac{k T}{q} \ln \left(\frac{A A^{*} T^{2}}{I_{s}}\right)
$$

The ideality factor is determined from the slope of the linear region of the plot of natural log of forward current versus forward bias voltage and is given by

$$
n=\frac{q}{k T}\left(\frac{d V}{d(\ln I)}\right)
$$

Typical forward bias $I-V$ characteristics of Au/n-Ge (100) Schottky contacts measured in the $140-300 \mathrm{~K}$ temperature range are shown in Fig. 1. These curves indicate a very strong temperature dependence of the Schottky diodes. The $I-V$ characteristics deviate from ideality at low temperature $(140-200 \mathrm{~K})$, which is due to the effect of other current transport 
processes like generation-recombination of carriers in space charge region [14] and tunneling of electrons through the barrier. At temperatures above $220 \mathrm{~K}$, thermionic emission becomes the dominant process. We have performed the least squares fit of Eq. (1) to the linear part of the intermediate forward bias region of the measured $I-V$ plots (Fig. 1), by assuming that this region consisted of TE current [1]. From these fits, the experimental values of $\Phi_{B}$ and $n$ are determined.

$\Phi_{B}$ and $n$ plots as a function of temperature for the Au/n-Ge (100) SBDs are presented in Fig. 2. The SBH shows a decrease with decrease in temperature, while the ideality factor increase with decrease in temperature. This depicts that both parameters exhibit strong temperature dependence. This temperature dependence can be attributed to the discontinuities at the interface which may exist even for well controlled fabrication of samples [15]. Since current flow across a MS interface is a temperature activated process $[9,10,13,16]$, the current transport will be dominated by current flowing through nanometer scale interfacial patches of small regions with lower $\mathrm{SBH}$ and a larger ideality factor [13]. As a result of inhomogeneities, charge transport across the interface is no longer dominated by TE. Furthermore, many models have been devolved to explain the inhomogeneity in the barrier [9,17,18]. A potential fluctuation model was proposed by Tung [9] to explain the inhomogeneity in SBHs which show a larger deviation from the classical TE theory at low temperature. From the potential fluctuation model, at sufficiently low temperatures, a large number of patches may be present and consequently high current flowing through these patches. As the temperature increases, more electrons have sufficient energy to surmount the higher barrier $[7,15]$.

\subsection{Analysis of inhomogeneous barrier height}

In order to describe the abnormal behaviour, i.e. the decrease in the SBH with decrease in temperature, a spatial distribution of the SBH at the MS interface of Schottky contacts was 
suggested by Werner and Guttler [6,19] to be a Gaussian distribution $P\left(\Phi_{B}\right)$ with a standard deviation $\left(\sigma_{s}\right)$ around a mean $\operatorname{SBH}\left(\bar{\Phi}_{B}\right)$ as:

$$
P\left(\Phi_{B}\right)=\frac{1}{\sigma_{s} \sqrt{2 \pi}} \exp \left[-\frac{\left(\Phi_{B}-\bar{\Phi}_{B}\right)^{2}}{2 \sigma_{s}^{2}}\right]
$$

where $\frac{1}{\sigma_{s} \sqrt{2 \pi}}$ is the normalization constant of the Gaussian barrier distribution. The Gaussian distribution of the SBH yields the following expression for experimental apparent SBH at zero-bias [20,21]:

$$
\Phi_{B}=\bar{\Phi}_{B}(T=0)-\frac{q \sigma_{s 0}^{2}}{2 k T}
$$

where $\bar{\Phi}(T=0)$ and $\sigma_{s 0}$ are the mean SBH and its standard deviation at zero-bias $(V=0)$, respectively. The temperature dependence of $\sigma_{s 0}$ is usually small and can be neglected [22]. The variation of experimental apparent ideality factor with temperature in this model is given by [19]:

$$
\left(\frac{1}{n}-1\right)=-\rho_{2}+\frac{q \rho_{3}}{2 k T}
$$

where $\rho_{2}$ and $\rho_{3}$ are the voltage coefficients that depict the voltage deformation of the barrier height distribution.

The experimental $\Phi_{B} v s \frac{1}{2 k T}$ and $\left(\frac{1}{n}-1\right) v s \frac{1}{2 k T}$ plots obtained by means of data extracted from Fig. 1 are shown in Fig. 3.

The temperature dependence of the SBH Au/n-Ge (100) Schottky diodes (Fig. 3) depicts one Gaussian distribution with $\bar{\Phi}_{B}(T=0)=0.615 \mathrm{eV}$ and $\sigma_{s 0}=0.0858 \mathrm{eV}$ in the $140-300 \mathrm{~K}$. In this temperature range, the ideality factor shows some linearity with temperature in two regions. The voltage coefficients have been obtained as $\rho_{21}=-0.454$ and $\rho_{31}=-0.028$ in the $180-300 \mathrm{~K}$ range, $\rho_{22}=0.182$ and $\rho_{32}=-0.0437$ in the $140-180 \mathrm{~K}$ range. The standard 
deviation is a measure of barrier homogeneity. The lower value of $\sigma_{s 0}$ indicates a more homogenous SBH [22]. When comparing $\bar{\Phi}_{B}(T=0)$ and $\sigma_{s 0}$ parameters, it is seen that the standard deviation is $\approx 13 \%$ of the mean zero-bias $\mathrm{SBH}$. This value $\sigma_{s 0}$ is not small compared to $\bar{\Phi}_{B}(T=0)$ and this indicates large inhomogeneity at interface of Au/n-Ge (100) structures. Hence, this inhomogeneity and potential fluctuations affect low temperature $I-V$ characteristics.

To determine the barrier height in another way, Eq. (2) can be written as

$$
\ln \left(\frac{I_{s}}{T^{2}}\right)=\ln \left(A A^{*}\right)-\frac{q \Phi_{B}}{k T}
$$

The Richardson constant is usually determined from the intercept of $\ln \left(\frac{I_{s}}{T^{2}}\right) v s \frac{1000}{T}$ plot.

Fig. 4 shows the conventional energy variation of $\ln \left(\frac{I_{s}}{T^{2}}\right) v s \frac{1000}{T}$ (labeled $\left.\mathrm{y}_{1}\right)$ for $\quad \mathrm{Au} / \mathrm{n}-\mathrm{Ge}$ (100) Schottky diodes. The experimental data shown, fit asymptotically to a straight line at higher temperatures. Since the conventional Richardson plot deviates from linearity at low temperatures due the barrier inhomogeneity, it can be modified by combining Eq. (2) and (6) which yields

$$
\ln \left(\frac{I_{s}}{T^{2}}\right)-\left(\frac{q^{2} \sigma_{s 0}^{2}}{2 k^{2} T^{2}}\right)=\ln \left(A^{* *} A\right)-\frac{q \bar{\Phi}_{B}}{k T}
$$

Fig. 4 shows the modified $\ln \left(\frac{I_{s}}{T^{2}}\right)-\left(\frac{q^{2} \sigma_{s 0}^{2}}{2 k^{2} T^{2}}\right)=\ln \left(A^{*} A\right)-\frac{q \bar{\Phi}_{B}}{k T}$ plot (labeled $\mathrm{y}_{2}$ ) for $\mathrm{Au} / \mathrm{n}-\mathrm{Ge}$ (100) Schottky diodes. The modified plot gives $\bar{\Phi}_{B}(T=0)=0.639 \mathrm{eV}$ and modified Richardson constant, $A^{* *}=1.37 \mathrm{Acm}^{-2} \mathrm{~K}^{-2}$. The value of $\bar{\Phi}_{B}(T=0)$ has almost the same value as the mean $\mathrm{SBH}$ obtained from $\Phi_{B} v s \frac{1}{2 k T}$ plot at higher temperatures in Fig. 3 . The 
modified Richardson constant is much smaller than the reported of $50 \mathrm{Acm}^{-2} \mathrm{~K}^{-2}$ [23]. This may be due to greater inhomogeneities at the interface.

\section{Summary and conclusions}

The $I-V-T$ characteristics of Au/n-Ge (100) Schottky contacts fabricated using the resistive evaporation system were measured in the $140-300 \mathrm{~K}$ range. While the zero-bias $\mathrm{SBH}\left(\Phi_{B}\right)$ increases with increasing temperature, the ideality factor $n$ decreases with increasing temperature. This observation has been attributed to barrier inhomogeneities explained by assuming a Gaussian distribution of SBHs due to barrier inhomogeneities that prevail at interface. In order to obtain evidence of a Gaussian distribution of SBHs, we have plotted $\Phi_{B}$ versus $\frac{1}{2 k T}$ graphs, and from which the values of $\bar{\Phi}_{B}(T=0)=0.615 \mathrm{eV}$ and $\sigma_{s 0}=0.0858$ $\mathrm{eV}$ for mean $\mathrm{SBH}$ and standard deviation, respectively, have been obtained. In the modified $\ln \left(\frac{I_{s}}{T^{2}}\right)-\left(\frac{q^{2} \sigma_{s 0}^{2}}{2 k^{2} T^{2}}\right) v s \frac{1000}{T}$ plot, the values of $\bar{\Phi}_{B}(T=0)$ and $A^{* *}$ were obtained as $0.639 \mathrm{eV}$ and $1.37 \mathrm{Acm}^{-2} \mathrm{~K}^{-2}$, respectively.

\section{Acknowledgement}

The authors would like to thank the South African National Research Foundation for financially supporting the research. 


\section{References}

[1] E.H. Rhoderick, R.H. Williams, Metal-Semiconductor Contacts, Claredon Press, Oxford University Press, Oxford, (1988) 19.

[2] V. Janardhanam, A. Ashok Kumar, V. Rajagopal Reddy, P. Narasimha Reddy, J. Alloys Compd 485 (2009) 467.

[3] E. Gur, S. Tuzemen, B. Kolic, C. Coskun, J. Phys. Condens. Matter. 19 (2007) 1966206.

[4] I. Dokme, S. Altindal, Semicond. Sci. Technol. 21 (2006)1053.

[5] S. Karatas, S. Altindal, M. Cakar, Physica B 357 (2005) 386.

[6] J.H. Werner, H.H. Güttler, J. Appl. Phys. 73 (1993) 1315.

[7] F.E. Cimili, M. Sağlam, H. Efeoğlu, A. Türüt, Physica B 404 (2009) 344.

[8] R. F. Schmitsdorf, T.U. Kampen, W. Mönch, J. Vac. Sci. Technol. B 15 (1997) 1221.

[9] R.T. Tung, Phys. Rev. B 45 (1992) 13509.

[10] R.T. Tung, J.P. Sullivan, F. Schrey, Mater. Sci. Eng. B 14 (1992) 266.

[11] S. Chand, J. Kumar, Appl. Phys. A 63 (1996) 171.

[12] S. Chand, Semicond. Sci. Technol. 17 (2002) 136.

[13] Ö. Faruk Yüksel, Physica B 404 (2009) 1993.

[14] S.M. Sze, Physics of Semiconductor Devices, $2^{\text {nd }}$ ed., John Wiley \& Sons, New York, 1981.

[15] M.B. reddy, A.A. Kumar, V. Jamardhanam, V.R. Reddy, P.N. Reddy, Current. Appl. Phys. 9 (2009) 972.

[16] A. Gümüş, A. Türüt, N. Yalçin, J. Appl. Phys. 91 (2002) 245.

[17] J.D. Levine, J. Appl. Phys. 42 (1971) 3991.

[18] F.A. Padovani, R. Stratton, Solid-State Electron. 9 (1996) 695.

[19] J.H. Werner, H.H. Güttler, J. Appl. Phys. 69 (1991) 1522.

[20] Y.P. Song, R.L. Van Meirhaeghe, W.H. Laflere, F. Cardon, Solid-State Electron. 29 
(1986) 633.

[21] S. Chand, S. Bala, Appl. Surf. Sci. 252 (2005) 358.

[22] V. Janardhanam, A.A. Kumar, V.R. Reddy, P.N. Reddy, J. Alloys Cmpd 485 (2009) 467.

[23] H.B. Yao, C.C. Tan, S.L. Liew, C.T. Chua, R. li, R.T.P. Lee, S.J. Lee, D.Z. Chi, International Workshop on Junction Technology Proc. (2005) 85. 


\section{Figure captions}

Fig. 1. Experimental forward-bias current-voltage characteristics of a Au/n-Ge (100) Schottky contact in the temperature range $140-300 \mathrm{~K}$.

Fig. 2. Temperature dependence of ideality factor and barrier height for Au/n-Ge (100) Schottky contact in the temperature range $140-300 \mathrm{~K}$.

Fig. 3. Temperature dependence of barrier height $\left(\Phi_{B}\right)$ and ideality factor $\left(\begin{array}{ll}1 / \mathrm{n} & 1) \text { for }\end{array}\right.$ Au/n-Ge (100).

Fig. 4. Richardson's plot of $\ln \left(\frac{I_{s}}{T^{2}}\right)$ versus $\frac{1000}{T}$ and modified $\ln \left(\frac{I_{s}}{T^{2}}\right)-\left(\frac{q^{2} \sigma_{s 0}^{2}}{2 k^{2} T^{2}}\right)$ versus $\frac{1000}{T}$. 
Fig. 1.

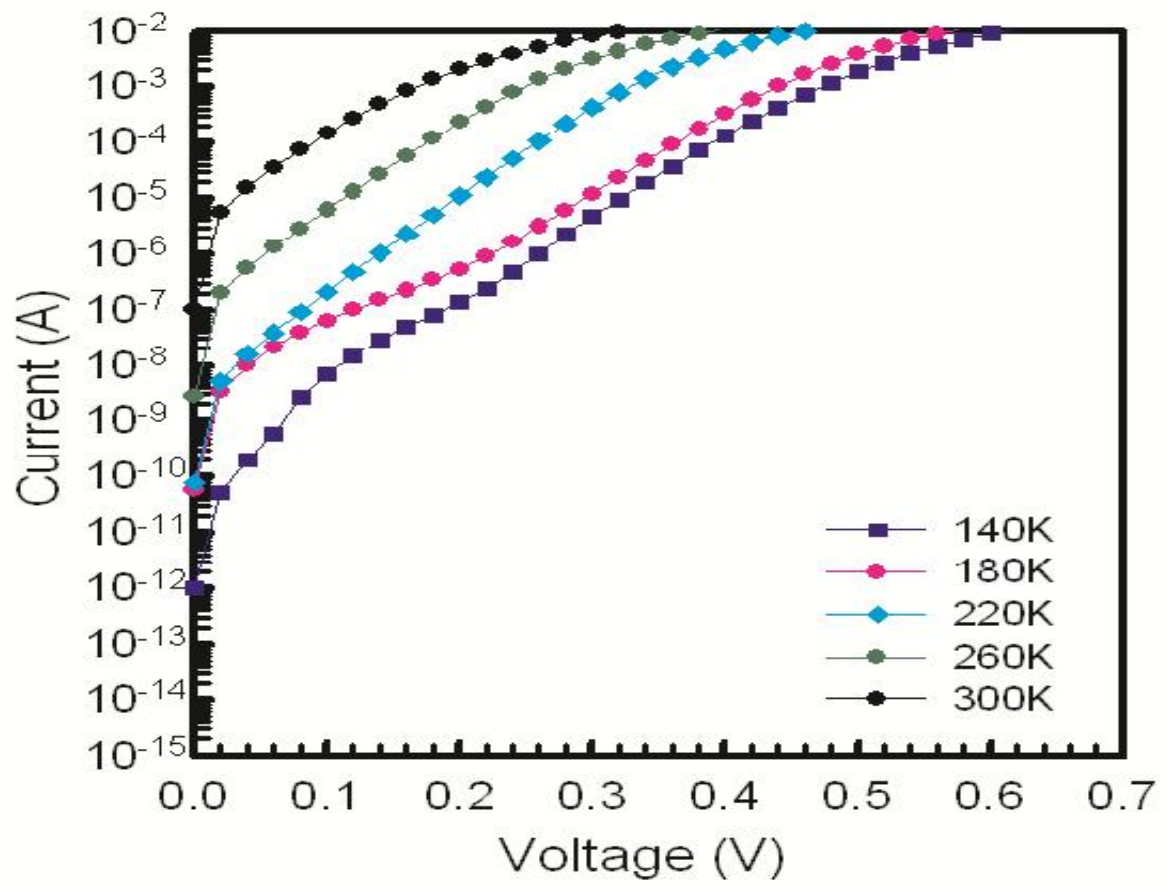


Fig. 2

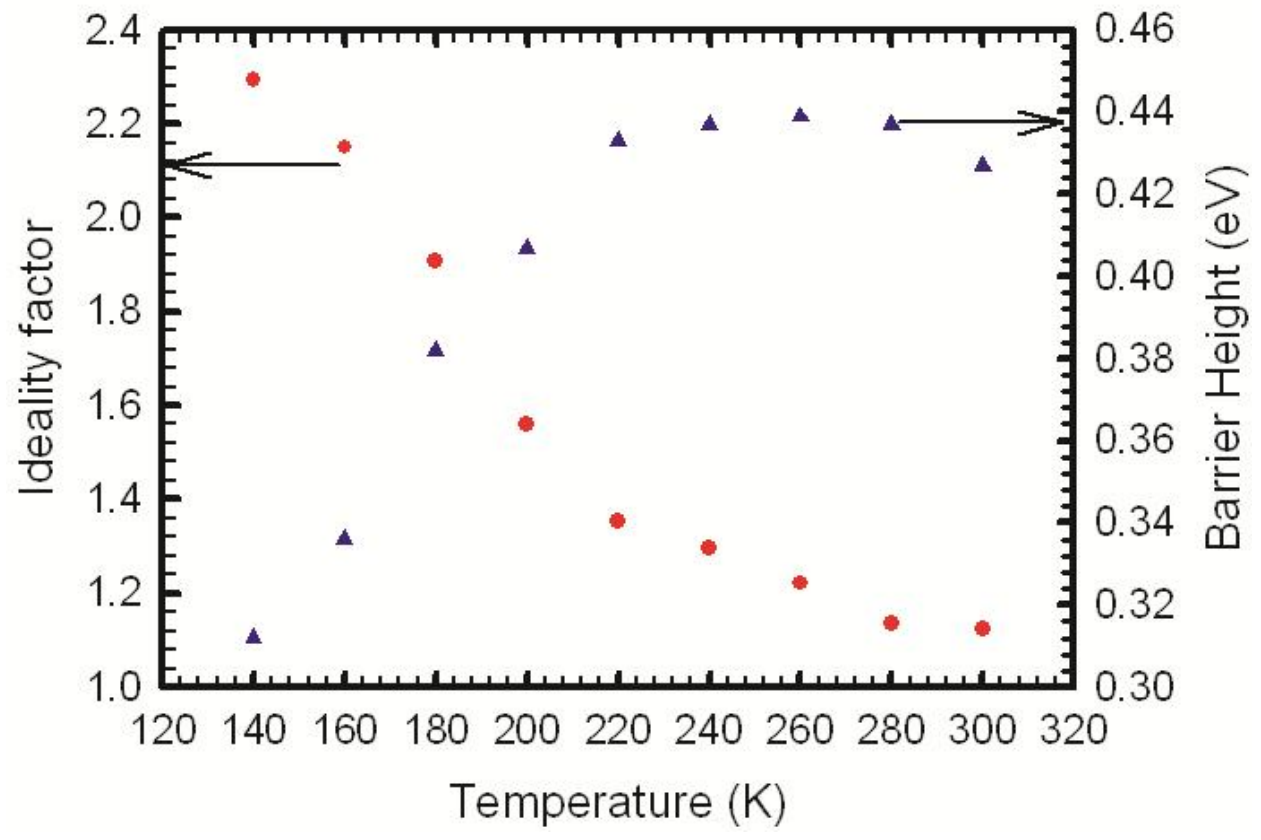


Fig. 3.

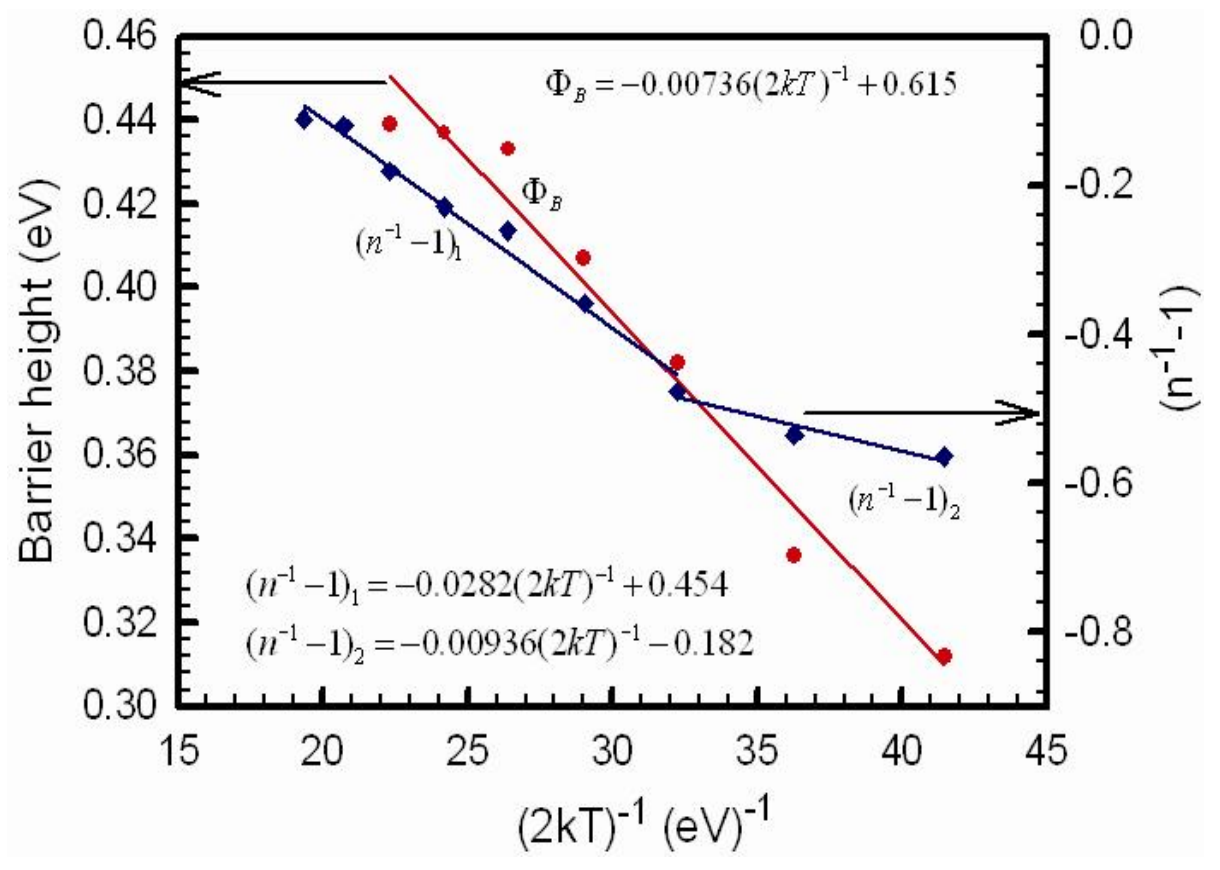


Fig. 4.

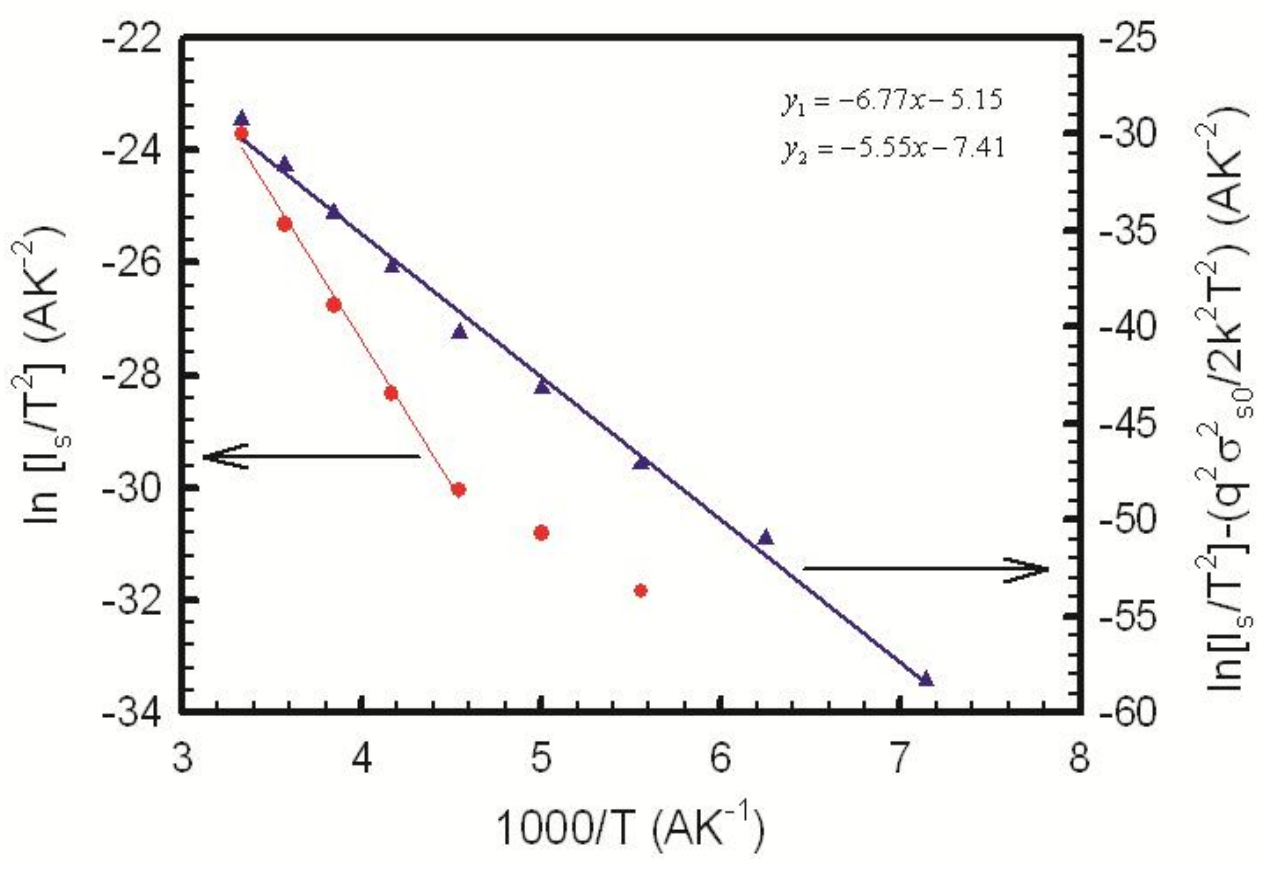

